\title{
Correspondence
}

\section{Acute respiratory distress syndrome}

Dear Sir,

The paper by E. Smith and I. Gordon "An Index to Predict Outcome in ARDS" [1] is of interest because of the good discrimination between survivors and non-survivors, although in a relatively small number of patients. It seem unfortunate, however, that they chose to use two factors in their score which are affected by therapy.

Both $\mathrm{AaDO}_{2}$ and $\Delta$ Paw are affected by therapeutic modalities determined by the clinician. $\mathrm{AaDO}_{2}$ is widely recognised as being very dependent on both PEEP and $\mathrm{FIO}_{2}$, and very different values may be obtained in the same patient with different levels of PEEP and $\mathrm{FIO}_{2}$. Similarly, the $\Delta$ Paw will vary according to the tidal volume (Vt) selected. For any given increase in Vt, there will be a greater increase in peak airway pressure (Paw) in a poorly compliant lung than in a normal lung, and so $\Delta \mathrm{Paw}$ will increase with increasing Vt.

Thus, two of the three factors used to determine the prognostic score can vary widely in the same patient depending on the ventilatory parameters selected. The score presumably correlates with outcome in the author's unit because of fairly standard and constant practices in relation to selection of $\mathrm{FIO}_{2}, \mathrm{PEEP}$ and $\mathrm{Vt}$ in given clinical circumstances, but one would expect that widely discrepant results would be obtained for the same patient by different clinicians in other units. For example, some clinicians routinely use $5 \mathrm{~cm} . \mathrm{H}_{2} \mathrm{O}$ PEEP in most patients with ARDS requiring ventilation, even at relatively low $\mathrm{FIO}_{2}$, and others use increasing PEEP until Qs/Qt is reduced to $15 \%$. In relation to Paw, our own practice is to reduce $\mathrm{Vt}$ in order to limit peak airway pressure in patients with severe ARDS, allowing $\mathrm{PaCO}_{2}$ to rise, because we believe that high Paw may be harmful.

A prognostic score should ideally predict the same outcome anywhere in the world for the same patient, regardless of such variations in clinician-selected variables. Only then will it be truly useful in allowing clinical decisions to be made, and in allowing comparison of outcome using different therapies. Thus it would seem preferable to use an index of oxygenation which is independent of ventilatory factors, such as $\mathrm{PaO}_{2}$ (or $\mathrm{Qs} / \mathrm{Qt}$ ) on $60 \% \mathrm{O}_{2}$ and $5 \mathrm{~cm}$.
$\mathrm{H}_{2} \mathrm{O}$ PEEP (as was used in the multicentre ECMO trial), [2] and a pressure-related index such as compliance over a stated volume range, which is less volume dependent than Paw.

Both the $\mathrm{FIO}_{2}$ (through oxygen toxicity), and the Vt (by affecting the peak airway pressure) may in fact affect the outcome in an individual patient. Unfortunately, however, this score will not allow survival comparisons in a clinical trial between managements using different approaches to $\mathrm{FIO}_{2}$, PEEP and Vt, as the score is itself dependent on these factors. The use of the Paw in a prognostic score in fact begs the question of whether high Paw is associated with poor outcome simply because it reflects poor lung compliance and is a bad prognostic sign, or because high Paw itself results in further progressive lung damage culminting in death, as suggested recently by Gattinoni and others $[3,4]$ and by a number of animal studies.

Finally, several recent studies have shown the major mortality in ARDS of moderate severity to be caused by sepsis or the underlying illness, not respiratory failure, and it must be questionable how accurate such a respiratory orientated score would be in predicting mortality in other groups of patients.

Your faithfully

K. G. Hickling

\section{References}

1. Smith P, Gordon I (1986) An index to predict outcome in adult respirtory distress syndrome. Intensive Care Med 12:86

2. Zapol WM, Snider MP, Hill JD et al. (1979) Extracorporeal membrane oxygenation in severe acute respiratory failure -- a randomized prospective study. J Am Med Assoc 242:2193

3. Gattinoni L, Agostoni A, Pesenti A et al. (1980) Treatment of acute respiratory failure with low frequency positive pressure ventilation and extracorporeal removal of $\mathrm{CO}_{2}$. Lancet $2: 292$

4. Hickling $\mathrm{K}$ (1986) Extracorporeal $\mathrm{CO}_{2}$ removal in severe adult respiratory distress syndrome. Anaesth Intensive Care 14:46

Dr. Keith G. Hickling, Department of Intensive Care, Christchurch Hospital, Christchurch, New Zealand 